\title{
Kinematical $x$-ray standing waves for crystal structure investigations
}

\author{
M. Tolkiehn and D. V. Novikov* \\ Hamburger Synchrotronstrahlungslabor HASYLAB at Deutsches Elektronen-Synchrotron DESY, Notkestraße 85, D-22603 Hamburg,
} Germany

S. S. Fanchenko

RRC Kurchatov Institute, Kurchatov Square 1, Moscow 123182, Russia

(Received 14 June 2004; revised manuscript received 29 November 2004; published 6 April 2005)

\begin{abstract}
A theoretical and experimental investigation of $\mathrm{x}$-ray standing wave formation in crystals under weak diffracted beam conditions is presented. The standing wave phase dependence is described using the kinematical approximation and is valid at large deviations from the Bragg angle for nonperfect crystals as well as for ideal crystals. This formalism was successfully applied to experimental data from perfect $\mathrm{GaAs}$ and $\mathrm{CaF}_{2} / \mathrm{Si}$ and from mosaic $\mathrm{Cu}_{3} \mathrm{Au}$ crystals. Based on these results, a novel method for kinematical x-ray standing wave analysis of nonperfect crystals is proposed.
\end{abstract}

DOI: 10.1103/PhysRevB.71.165404

PACS number(s): 68.49.Uv, 61.10.Dp, 61.43.-j

\section{INTRODUCTION}

The majority of x-ray scattering methods are based on measuring the scattered wave intensity in the far field. In contrast, inside the object, the incident wave coherently interferes with the scattered wave and gives rise to an x-ray standing wave field. In case of Bragg scattering in crystals, the phase of the standing wave is directly dependent on the deviation from the exact Bragg conditions. This effect is used in the X-ray standing wave (XSW) method: knowing the variation of the standing wave field given by dynamical $\mathrm{x}$-ray diffraction theory ${ }^{1}$ and monitoring the field intensity at atom positions through a secondary radiation, one can detect the positions of emitting atoms in the crystal unit cell ${ }^{2}$ or analyze the crystal structure. ${ }^{3}$

The common limitation of the conventional XSW method for crystals is the necessity of dynamical diffraction for standing wave formation, which requires a perfect or nearly perfect crystalline lattice, at least along the chosen directions. $^{4}$

An application of the XSW method to nonperfect crystals was so far limited to special techniques. The normal incidence XSW method ${ }^{5,6}$ (NIXSW) makes use of the drastic widening of the dynamical reflection curves at Bragg angles near $90^{\circ}$. This backscattering geometry imposes some limitations on reflections and energies available for investigation. Another effective approach is using micro beams to limit the diffraction volume to a small sufficiently perfect part of the studied material. ${ }^{7}$ Finally, XSW was used to define polarity or impurity position in thin films. ${ }^{8}$ Due to the low thickness of the films under investigation, the diffraction can be described in the frame of kinematical approximation.

The kinematical approximation can always be used, when the elastically scattered wave is weak compared to the incident. This happens in ideal mosaic crystals. In real samples there can always exist a dynamical scattering component, which makes the analysis in the vicinity of the Bragg angle rather cumbersome. ${ }^{9}$ However at large deviations the kinematical theory is always valid regardless of the lattice perfection. In this work, we theoretically analyze the phase ef- fects of the standing wave field in bulk single crystals far away from Bragg diffraction conditions. On this basis we propose a new method of kinematical $x$-ray standing waves and present a first experimental feasibility study. The method can be used for the determination of dopant positions in nonperfect crystals or for the investigation of surface layers on nonperfect crystalline substrates.

\section{THEORY}

Let us first consider the Bragg diffraction from a perfect crystal far from the Bragg angle. In this case, the scattered wave can be correctly described by the dynamical theory of $\mathrm{x}$-ray diffraction. ${ }^{1}$ The ratio of the electrical field amplitudes of incident and reflected beam is given by the parameter

$$
\alpha=-\frac{|P| \sqrt{|b|}}{P} \frac{\left(F_{\mathbf{H}} F_{\mathbf{H}}^{-}\right)^{1 / 2}}{F_{\mathbf{H}}^{-}}\left(\eta \pm\left(\eta^{2}-1\right)^{1 / 2}\right),
$$

where $\mathbf{H}$ is the reciprocal lattice vector and $F_{\mathbf{H}}$ the structure factor corresponding to the reflection. The dimensionless parameter $\eta$ is proportional to the deviation $\Delta \theta$ from the Bragg angle $\theta_{B}, P$ is the polarization factor, and $b$ is the asymmetry factor. ${ }^{10}$

For deviations from the Bragg angle which are large compared to the reflection half-width, the amplitude of the scattered wave becomes small and the kinematical approximation can be applied. Introducing a small parameter $x(\Delta \theta)$ $=1 / \eta(\Delta \theta)$ and approximating Eq. (1) by the first order Taylor polynomial in $x$ gives:

$$
\begin{aligned}
& \alpha \approx-\frac{|P| \sqrt{|b|}}{P} \frac{\left(F_{\mathbf{H}} F_{\mathbf{H}}^{-}\right)^{1 / 2}}{F_{\mathbf{H}}^{-}} \frac{1}{2} x \\
& =\frac{P \Gamma F_{\mathbf{H}}}{2 \Delta \theta \sin \left(2 \theta_{B}\right)+\Gamma F_{0} \frac{1-b}{b}}
\end{aligned}
$$

In the region where incident and reflected beams overlap, they interfere coherently and form a standing wave field with the intensity 


$$
\begin{gathered}
\frac{I(\mathbf{r})}{\left\|\mathbf{E}_{0}\right\|^{2}}=1+2 P \operatorname{Re}\left(\alpha e^{-i \mathbf{H} \cdot \mathbf{r}}\right)+\frac{|\alpha|^{2}}{\approx 0} \\
\approx 1+P^{2} \Gamma \operatorname{Re}\left(\frac{F_{\mathbf{H}} e^{-i \mathbf{H} \cdot \mathbf{r}}}{\Delta \theta \sin \left(2 \theta_{B}\right)+\Gamma F_{0} \frac{1-b}{2 b}}\right) .
\end{gathered}
$$

Using $F_{0}=F_{0}^{\prime}+i F_{0}^{\prime \prime}$, Eq. (3) can be simplified to

$$
\frac{I(\mathbf{r})}{\left\|\mathbf{E}_{0}\right\|^{2}}=1+\frac{P^{2} \Gamma}{\sin 2 \theta_{B}}\left(\frac{\Delta \theta^{\prime} \operatorname{Re}\left(F_{\mathbf{H}} e^{-i \mathbf{H} \cdot \mathbf{r}}\right)}{\Delta \theta^{\prime 2}+\delta^{2}}+\frac{\delta \operatorname{Im}\left(F_{\mathbf{H}} e^{-i \mathbf{H} \cdot \mathbf{r}}\right)}{\Delta \theta^{\prime 2}+\delta^{2}}\right) .
$$

Here $\quad \Delta \theta^{\prime}=\Delta \theta+\Gamma F_{0}^{\prime}(1-b) /\left(2 b \sin 2 \theta_{B}\right) \quad$ and $\quad \delta=\Gamma F_{0}^{\prime \prime}(1$ $-b) /\left(2 b \sin 2 \theta_{B}\right)$. For large deviations from Bragg conditions where $1 / \Delta \theta^{\prime 2} \ll 1 / \Delta \theta^{\prime}$ and far away from absorption edges where $F_{0}^{\prime \prime}$ is small, the second term in Eq. (4) can be neglected.

For a crystal structure with $n$ fluorescent atoms at positions $\mathbf{r}_{j}$ the total fluorescence yield is then given by

$$
\begin{aligned}
& Y \propto n+\sum_{j=1}^{n} \frac{P^{2} \Gamma}{\sin 2 \theta_{B}} \frac{\Delta \theta^{\prime}\left|F_{\mathbf{H}}\right| \cos \left(\mathbf{H} \cdot \mathbf{r}_{j}-\arg F_{\mathbf{H}}\right)}{\Delta \theta^{\prime 2}+\delta^{2}} \\
& \propto 1+\frac{P^{2} \Gamma}{\sin 2 \theta_{B}} \frac{\Delta \theta^{\prime}\left|F_{\mathbf{H}}\right| f_{c} \cos \left(2 \pi \Phi_{c}-\arg F_{\mathbf{H}}\right)}{\Delta \theta^{\prime 2}+\delta^{2}},
\end{aligned}
$$

where the coherent fraction $f_{c}$ and the coherent phase $\Phi_{c}$ are defined as the amplitude and the phase of the Fourier coefficient of sublattice formed by the fluorescing atoms. ${ }^{2}$

In the case of ideal mosaic crystals, the equation for the reflectivity can be obtained by direct calculation in the frame of kinematical theory. The integration of the well-known Thomson scattering formula ${ }^{11}$ must be carried out taking into account the near field terms and the curvature of the spherical waves, as the field is calculated inside the crystal. The result after integration over the sample volume yields Eq. (4) with $\delta=0$, since absorption is neglected in the classical kinematical approximation. By convolving Eq. (4) with a Lorentzian mosaic spread function of width $\sigma$, one obtains:

$$
\frac{I(\mathbf{r})}{\left\|\mathbf{E}_{0}\right\|^{2}}=1+\frac{P^{2} \Gamma}{\sin 2 \theta_{B}} \frac{\Delta \theta^{\prime} \operatorname{Re}\left(F_{\mathbf{H}} e^{-i \mathbf{H} \cdot \mathbf{r}}\right)}{\Delta \theta^{\prime 2}+\sigma^{2}} .
$$

The parameter $\sigma$ can be determined from the half-width of the measured reflectivity curve. The result for the fluorescence yield is the same as in Eq. (5) where $\delta$ is replaced by $\sigma$. Details of the calculations will be published elsewhere.

At weak diffraction conditions, the form of the fluorescence yield curve depends on the dimensionless parameter

$$
\Upsilon_{c}=\left|F_{\mathbf{H}}\right| f_{c} \cos \left(2 \pi \Phi_{c}-\arg F_{\mathbf{H}}\right),
$$

which can be obtained by fitting experimental data. In order to determine both $f_{c}$ and $\Phi_{c}$, at least two values of $\Upsilon_{c}$ measured at different energies are needed. Varying the energy of the incident beam changes the phase of the structure factor $\arg F_{\mathbf{H}}$ and leads to a simple set of equations from which both $f_{c}$ and $\Phi_{c}$ can be calculated. The vicinity of absorption

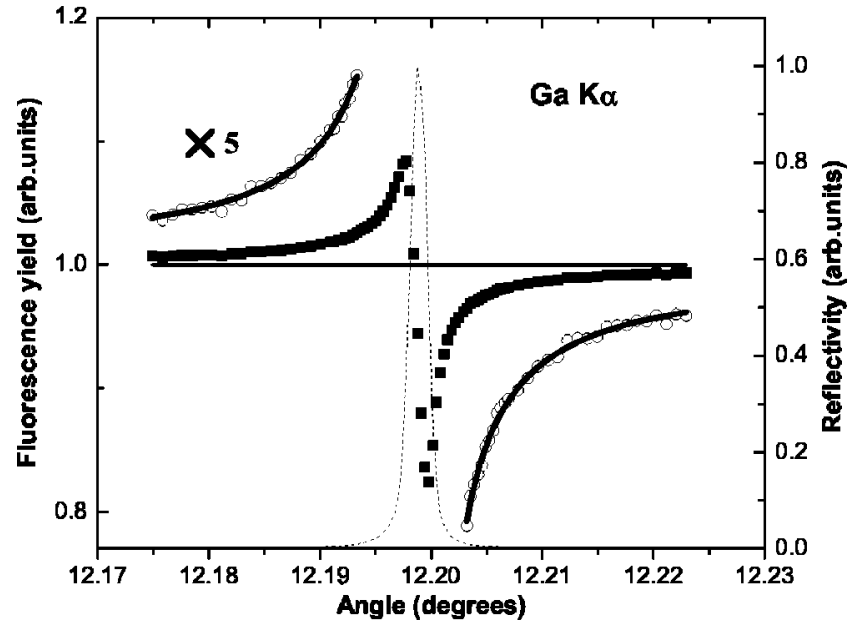

FIG. 1. GaAs(002) Bragg reflection at $10.39 \mathrm{keV}: \mathrm{Ga} K_{\alpha}$ fluorescence yield (squares) and reflectivity curve (dashed line). The data points used for the kinematical data evaluation (circles) and the theoretical curve (solid line) are shown magnified by factor 5 . The statistical errors of the measurement are better then $0.05 \%$ at all points.

edges, where the phase shift of $F_{\mathbf{H}}$ is largest, has to be avoided in order to comply with the approximation of Eq. (4).

\section{EXPERIMENTS}

To demonstrate the applicability of the derived equations XSW measurements on three different samples were performed. First a single crystalline $\mathrm{GaAs}(001)$ wafer was used to show the sensitivity to structure factor phase variations. Next an epitaxial $\mathrm{CaF}_{2} / \mathrm{Si}$ heterostructure was used to compare the results of the kinematical XSW and conventional, dynamical XSW evaluation. Finally the method was applied to a mosaic $\mathrm{Cu}_{3} \mathrm{Au}$ single crystal.

The experiments were carried out at HASYLAB at the beamlines BW1 and CEMO using a symmetrically cut double-crystal $\mathrm{Si}(111)$ monochromator. The fluorescence was measured with an energy dispersive silicon drift detector (Röntec XFlash). For the reflectivity an unbiased PN diode was used.

\section{A. Structure factor phase determination: GaAs (002)}

For the (002) reflection and the origin of the lattice unit cell chosen at a Ga atom, the factor $e^{-i \mathbf{H} \cdot \mathbf{r}_{j}}$ for the Ga fluorescence yield in Eq. (5) is always equal to one. Neglecting the Debye-Waller factor, one obtains $Y_{c}=\operatorname{Re} F_{(002)}$. If $\left|F_{(002)}\right|$ is known, for instance from the Bragg reflection intensity, one can directly determine the phase of the (002) structure factor.

Figure 1 shows a typical fluorescence yield curve for the $\mathrm{Ga} K_{\alpha}$ line. The datapoints where the reflectivity is $<1 \%$ were used for the kinematical evaluation. These parts are presented magnified together with the best fit curve. The theoretical and experimental curves show a good agreement. The values of $Y_{c}$ obtained from the experiment at incident 


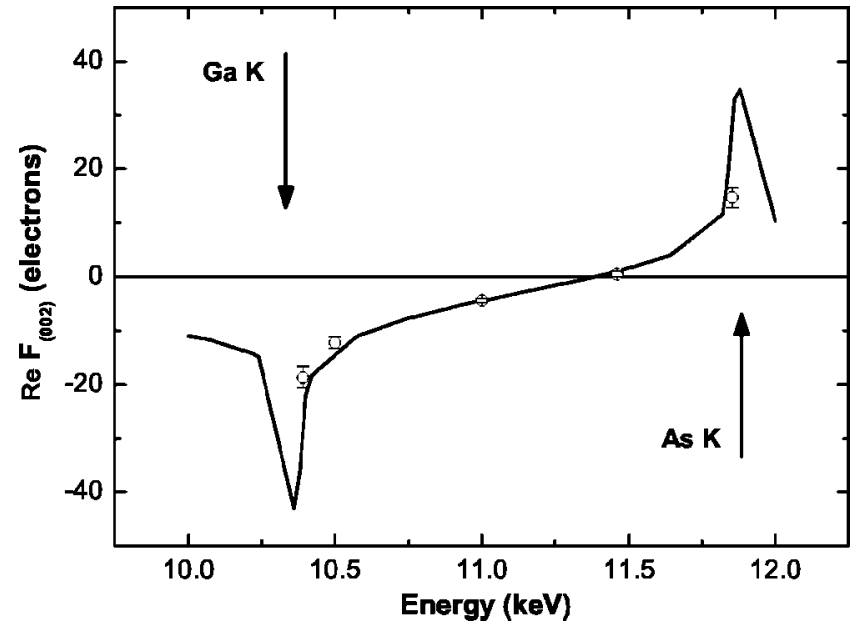

FIG. 2. Real part of $F_{(002)}$ measured with kinematical XSW (dots) and theoretically calculated (line) using the Henke database (Ref. 12). The arrows mark the Ga and As absorption edges.

beam energies in the range between $10.39 \mathrm{keV}$ and $11.85 \mathrm{keV}$ and the corresponding theoretical curve (Fig. 2) in the central region between the $\mathrm{Ga} K$ and As $K$ absorption edges coincide within the experimental error. This energy range is most suitable for the determination of both $f_{c}$ and $\Phi_{c}$. The deviation in the vicinity of the Ga and As absorption edges may be attributed to the limited applicability of the Henke database ${ }^{12}$ at these energies. From the datasets at $11 \mathrm{keV}$ and $11.46 \mathrm{keV}$ one obtains $f_{c}=0.91 \pm 0.022$ and $\Phi_{c}$ $=-0.005 \pm 0.004$. The theoretical values, taking into account thermal vibrations, are $f_{c}=0.96$ and $\Phi_{c}=0$.

\section{B. Structure determination of $\mathrm{CaF}_{2} / \mathrm{Si}(111)$}

Even if the experimental conditions do not allow the observation of a sufficient phase shift, a single energy measurement can provide valuable information. This can be seen from kinematical XSW measurements carried out at a 18 monolayers $\mathrm{CaF}_{2}$ film grown by molecular beam epitaxy on Si(111). ${ }^{13}$ Two structural models, which differ by the interface bond length, are proposed for this system. ${ }^{14}$ The difference is large enough to cause different signs of $Y_{c}$ and the knowledge of $f_{c}$ is not necessary. The incident beam energy of $8 \mathrm{keV}$ chosen for the experiment lies far above the absorption edges of $\mathrm{Si}$ and $\mathrm{Ca}$. The X-ray standing wave field is formed by the perfect $\mathrm{Si}$ substrate and extends across the thin layer. The Bragg scattering from the layer itself can be neglected. Reflectivity and fluorescence were measured in the range of $0.06^{\circ}$ around the Bragg angle, the half-width of the $\mathrm{Si}(111)$ reflection being $0.0027^{\circ}$. The $\mathrm{Ca} K_{\alpha}$ fluorescence yield curve is shown in Fig. 3. As in the GaAs experiment, the kinematical theory was applied for the evaluation of the tails of the curve. The central region has been also used for the standard XSW data evaluation based on the dynamical theory. ${ }^{2}$ Although the statistical quality of the data is lower in the tails of the fluorescent yield curves, the kinematical XSW treatment provides a $Y_{c}$ value for the Ca atoms, which is in good agreement with the results of the conventional XSW evaluation (Table I). The measured $Y_{c}$ value is consistent

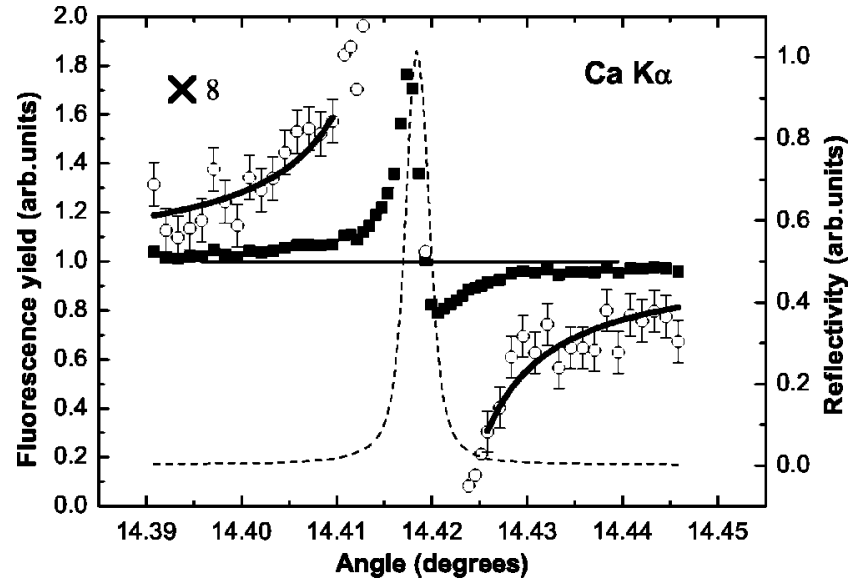

FIG. 3. $\mathrm{CaF}_{2} / \mathrm{Si}(111)$ : $\mathrm{Ca}$ fluorescence yield (squares) and reflectivity curve (dashed line). The data points used for the kinematical data evaluation (circles) and the theoretical curve (solid line) are shown magnified by factor 8 .

with position of the $\mathrm{Ca}$ atoms predicted by the long interface model. ${ }^{14}$

The single energy kinematical XSW evaluation cannot provide the full information on position and coherent fraction of the epitaxial film atoms but is fully sufficient to distinguish between the existing models. The precision of the kinematical method for this object proves to be comparable with that of the conventional XSW, even though the counting statistics in the central region is better than that in the tails.

\section{Mosaic crystals: $\mathrm{Cu}_{3} \mathrm{Au}$}

For nonperfect, mosaic single crystals the reflectivity curve is drastically broadened and the dynamical theory is not applicable. The fast variation of the fluorescence yield in the vicinity of the Bragg angle, produced by scattering in periodic structures, ${ }^{15}$ is also smeared out by the mosaic spread and cannot be used for evaluation. However, the broadening effects on the slowly varying tails of the curves are much smaller, so that the kinematical XSW approach is still feasible.

This is shown for a $\mathrm{Cu}_{3} \mathrm{Au}$ (001) mosaic single crystal with a mosaic spread of $\sigma=0.09^{\circ}$ [see Eq. (7)]. XSW measurements were performed at the (002) reflection for two different energies of the incident radiation: $9.2 \mathrm{keV}$ and

TABLE I. Comparison of the results for $\mathrm{CaF}_{2} / \mathrm{Si}(111)$ from kinematical and dynamical XSW measurements with the theoretical values for the long and the short interface models (Ref. 14). The theoretical values for $Y_{c}$ have been calculated with the measured value for $\mathrm{f}_{c}$.

\begin{tabular}{lccc}
\hline \hline & $\mathrm{f}_{c}$ & $\Phi_{c}$ & $\Upsilon_{c}$ \\
\hline kinematical XSW & & & $-40 \pm 1$ \\
dynamical XSW & $0.657 \pm 0.005$ & $0.53 \pm 0.002$ & $-39.7 \pm 0.3$ \\
long interface & & 0.46 & -38.6 \\
short interface & & 0.93 & 36.2 \\
\hline \hline
\end{tabular}



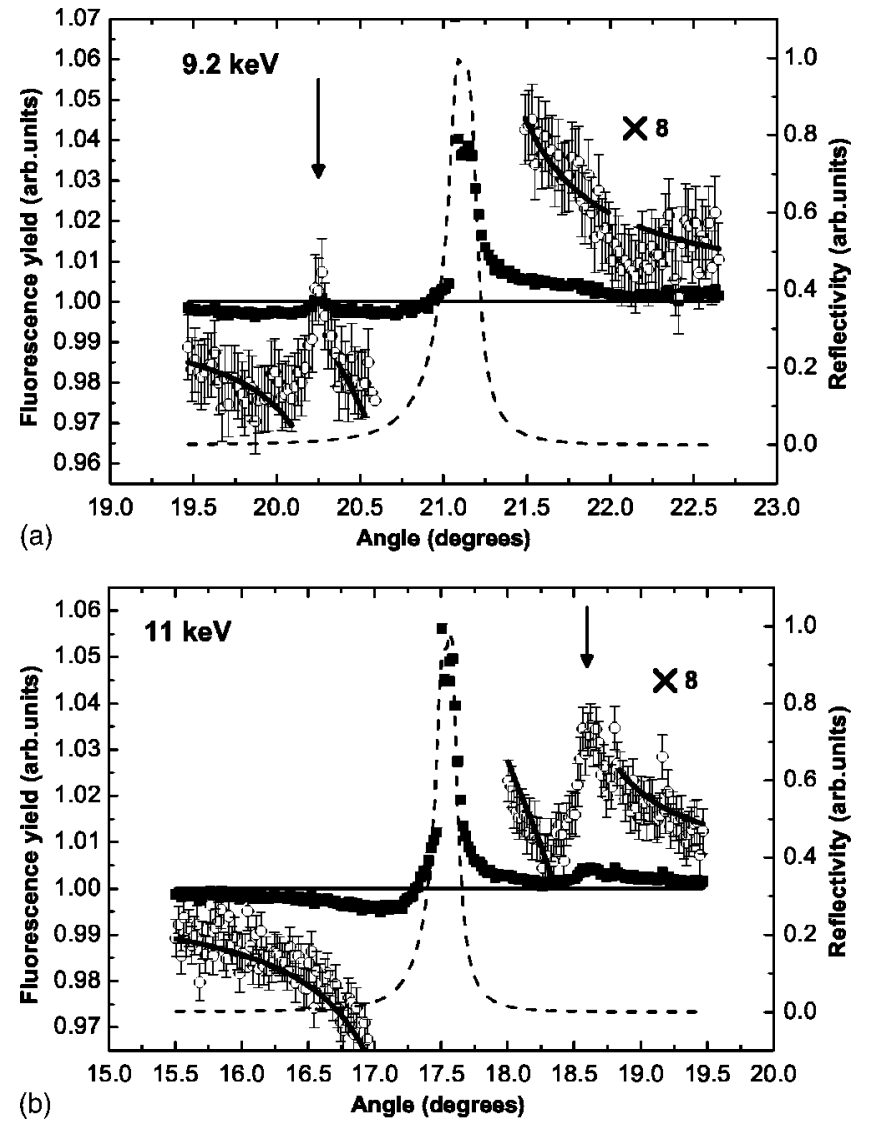

FIG. 4. $\mathrm{Cu}_{3} \mathrm{Au}(002)$ Bragg reflection at $9.2 \mathrm{keV}$ and $11 \mathrm{keV}: \mathrm{Cu}$ $K_{\alpha}$ fluorescence yield (squares) and reflectivity curve (dashed line). The data points used for the kinematical data evaluation (circles) and the theoretical fit curve (solid line) are shown magnified by factor 8. The arrows mark the fluorescence peaks from Bragg reflections other than (002). These angular ranges were excluded from evaluation.

$11 \mathrm{keV}$. Figure 4 shows the (002) reflectivity, the $\mathrm{Cu} K_{\alpha}$ fluorescence yield curves and the best fit. For both energies the curve is influenced by multiple reflections. Their effect was taken into account by excluding the data points in central regions of all reflection from the evaluation and by fitting the curve as a sum of multiple fluorescence yield curves. Since the angular distance between reflections was large interference effects as Umweganregung can be neglected. The results of the evaluation for the (002) reflection are shown in Table II. Knowing the $Y_{c}$ values and the coherent position in the $\mathrm{Cu}_{3} \mathrm{Au}$ fcc lattice, the coherent fraction of the copper atoms are obtained from Eq. (8).

The low measured values of the coherent fraction in mosaic crystals are determined not only by the local disorder in a sublattice, but also by displaced atoms in the block boundaries, twins and other strongly defected material regions. Besides, the standing wave field formed in a mosaic block spreads over its boundaries and penetrates the surrounding lattice. As the blocks are randomly displaced at atomic scales, the fluorescence yield coming from neighbouring blocks is out of phase and lowers the evaluated coherent fraction.
TABLE II. Experimental values of $Y_{c}$ for the $\mathrm{Cu}_{3} \mathrm{Au}(002)$ reflection and the $\mathrm{f}_{c}$ values calculated with Eq. (8). For both energies the obtained coherent fractions coincide within the experimental precision. The lattice origin was taken at the Au atoms, which corresponds to a coherent position of $\Phi_{c}=0$ for the $\mathrm{Cu}$ atoms. Theoretical $Y_{c}$ values for a perfect $\mathrm{Cu}_{3} \mathrm{Au}$ crystal with $\mathrm{f}_{c}=1$ are given for reference.

\begin{tabular}{cccc}
\hline \hline$E(\mathrm{keV})$ & $\Upsilon_{c}$ & $\Upsilon_{c, \text { perfect }}$ & $\mathrm{f}_{c}$ \\
\hline 9.2 & $91 \pm 2$ & 111.0 & $0.82 \pm 0.02$ \\
11 & $97 \pm 2$ & 116.5 & $0.83 \pm 0.02$ \\
\hline \hline
\end{tabular}

\section{DISCUSSION}

The physics of $\mathrm{x}$-ray standing wave field formation at large deviations from the Bragg angle and properties of mosaic crystals determine some special features of the described kinematical $x$-ray standing wave (KXSW) method.

In the conventional XSW method the shape of the fluorescence yield curve varies strongly with the position of the emitting atom in the unit cell. This is defined by the dynamical scattering conditions. Meanwhile, the shape of the tails, used in the KXSW, stays unchanged, and the emitting atom position influences only the amplitude and sign of the signal $\left[Y_{c}\right.$, Eq. (8)]. Strictly speaking, the KXSW does not require information of the shape of the fluorescence yield curves, but only of the amplitude difference on both sides of a Bragg reflection. The atoms lying on the diffraction planes will provide the maximal yield variation, and atoms in other positions will deliver weaker signals. Since the signal is measured relative to a constant background, the same counting statistic is necessary in order to determine $Y_{c}$ with a given precision independent of the emitting atom position in the cell.

The influence of mosaicity on the tails of the curves also stays weak independent of the phase, which is not the case in the vicinity of the Bragg angle. The shape variation of the curve due the mosaic spread does not complicate evaluation of signal difference on both sides of the Bragg angle and can be easily done following Eq. (7). In contrast, the effect of intermixing of standing wave fields from different crystallites cannot be avoided in mosaic samples and leads to an effective reduction of the measured coherent fraction values. However, its influence on the KXSW results will be negligible for a wide class of objects, where the emitting atoms reside in the field of one crystallite only, for example, as in the case of adsorbate atoms in surface science studies.

In a case of emitting atoms occupying multiple nonequivalent positions, the coherent fraction $f_{c}$ is reduced according to Eq. (5). Since for large deviation from exact Bragg conditions $f_{c}$ and $\Phi_{c}$ influence only the amplitude of the signal [see Eq. (8)], at least two measurements at different wavelengths are necessary to obtain both parameters, whereas for the conventional XSW method ${ }^{2}$ a single curve is sufficient for fitting both $f_{c}$ and $\Phi_{c}$. For the two energy KXSW measurements, the precision of $f_{c}$ and $\Phi_{c}$ depends only on the phase shift of the structure factor and the precision of $Y_{c}$. If no significant change of the structure factor 
phase can be achieved by energy variation, multiple linearly dependent reflections can be used to verify whether a single position model is valid [e.g. (111) and (333) as in Bedzyk et $a l .{ }^{16}$ ]. A single energy measurement can be also useful to make a choice between several existing models of atom arrangement, as in the $\mathrm{CaF}_{2} / \mathrm{Si}$ experiment.

Due to the wide angular range of the data which is necessary for the KXSW method the fluorescence yield can often be influenced by multiple beam reflection effect. In Fig. 4, these intervals are marked by arrows. Unlike in the dynamical scattering case the phases of the diffracted waves for different reflections are not coupled and the fluorescence yield represents a simple sum over all reflections. Evaluation of such data can be done as a multiple parameter fit with known coefficients for all reflection. In a general case, the reflections are noncoplanar, and the experiment requires an incident beam collimated in both planes. This can be easily achieved on third generation synchrotron sources. The precision of the KXSW results will grow with number of reflections included into evaluation. A maximal possible number would be achieved if the fluorescence yield data is collected for all angles of incidence on the hemisphere. The so obtained data set is equivalent to those used in x-ray atom resolving holography. ${ }^{17,18}$

The precision of KXSW on crystals with high lattice quality is of the same order as of dynamical XSW, as confirmed by the $\mathrm{GaAs}$ and $\mathrm{CaF}_{2} / \mathrm{Si}$ experiments. The conventional method is still more favorable for perfect crystals as one can define $f_{c}$ and $\Phi_{c}$ from one measurement. This is also valid for the normal incidence $\mathrm{XSW},{ }^{6}$ that can be applied to objects of lower quality. The NIXSW method stays in the frame of dynamical scattering conditions for high mosaicity values, but is bound to a limited number of reflections and energies.

Let us consider as an example the $\mathrm{Cu}_{3} \mathrm{Au}$ crystal with a mosaicity of $\sigma=0.09^{\circ}$ used in this work. In order to perform NIXSW measurements the dynamical reflection curve half- width should exceed the mosaic spread. This condition is fulfilled in the case of the (002) reflection, where backscattering is achieved at about $3.31 \mathrm{keV}$ and the halfwidth $0.55^{\circ}$ can easily accommodate the mosaicity. All higher order reflection curves appear to be too narrow. It will be therefore difficult to determine with NIXSW the emitting atom position in three dimensions or handle a multiple position case. KXSW measurements would provide an additional access to such problems. Besides, the possibility to freely choose the x-ray wavelength can bring advantages for in situ investigations in complicated environments as gas reactors or electrolytic cells.

\section{CONCLUSIONS}

The obtained results serve as a basis for a novel kinematical $x$-ray standing wave method for investigation of mosaic single crystals. In contrast to the established dynamical XSW method, it employs the standing waves far from the exact Bragg conditions and allows us to overcome constraints on the crystal quality imposed by dynamical scattering conditions. The method is complimentary to the conventional XSW and can open new possibilities in the investigation of natural minerals, metallic and organic single crystals, interface and surface processes. ${ }^{19}$ The KXSW theoretical approach can be also used for evaluation of holographic data from crystalline objects. ${ }^{20,21}$

\section{ACKNOWLEDGMENTS}

The authors are grateful to K. Hofmann and C.R. Wang of the IHW Institut für Halbleiterbauelemente und Werkstoffe at the University of Hannover for the preparation of the $\mathrm{CaF}_{2}$ sample and to V. Kaganer, P. Korecki, and G. Materlik for helpful discussions.
*Electronic address: Dmitri.Novikov@desy.de

${ }^{1}$ M. von Laue, Röntgenstrahlinterferenzen, 3rd ed. (Akademische Verlagsgesellschaft, Frankfurt am Main, 1960).

${ }^{2}$ J. Zegenhagen, Surf. Sci. Rep. 18, 202 (1993).

${ }^{3}$ M. J. Bedzyk and G. Materlik, Phys. Rev. B 32, 6456 (1985).

${ }^{4}$ L. Cheng, P. Fenter, M. J. Bedzyk, and N. Sturchio, Phys. Rev. Lett. 90, 255503 (2003).

${ }^{5}$ T. Ohta, Y. Kitajama, H. Kuroda, T. Takahashi, and S. Kikuta, Nucl. Instrum. Methods Phys. Res. A 246, 760 (1986).

${ }^{6}$ D. P. Woodruff, Prog. Surf. Sci. 57, 1 (1998).

${ }^{7}$ M. Drakopulous, J. Zegenhagen, A. Snigirev, I. Snigireva, M. Hauser, K. Eberl, V. Aristov, L. Shabelnikov, and V. Yunkin, Appl. Phys. Lett. 81, 2279 (2002).

${ }^{8}$ A. Kazimirov, N. Faleev, H. Temkin, M. Bedzyk, V. Dmitriev, and Y. Melnik, J. Appl. Phys. 89, 6092 (2001), and references $14,15,19$, and 20 therein.

${ }^{9}$ T. M. Sabine, in International Tables For Crystallography, edited by A. J. C. Wilson (Kluwer academic publishers, 1992), Vol. C, p. 530.

${ }^{10}$ B. W. Batterman and H. Cole, Rev. Mod. Phys. 36, 681 (1964).
${ }^{11}$ J. D. Jackson, Classical Electrodynamics, 2nd ed. (Wiley, New York, 1975).

${ }^{12}$ B. Henke, E. Gullikson, and J. Davis, At. Data Nucl. Data Tables 54, 181 (1993).

${ }^{13}$ C. R. Wang, B. H. Müller, and K. R. Hofmann, Thin Solid Films 410, 72 (2002).

${ }^{14}$ Y. Itoh, I. Takahashi, A. Ichimiya, J. Harada, and N. S. Sokolov, J. Cryst. Growth 166, 61 (1996).

${ }^{15}$ V. M. Kaganer, S. A. Stepanov, and R. Köhler, Physica B 221, 34 (1996).

${ }^{16}$ M. J. Bedzyk and G. Materlik, Phys. Rev. B 31, 4110 (1985).

${ }^{17}$ M. Tegze and G. Faigel, Europhys. Lett. 16, 41 (1991).

${ }^{18}$ T. Gog, P. M. Len, G. Materlik, D. Bahr, C. S. Fadley, and C. Sanchez-Hanke, Phys. Rev. Lett. 76, 3132 (1996).

${ }^{19}$ M. J. Bedzyk and L. Cheng, Rev. Min. Geochem. 49, 221 (2002).

${ }^{20}$ B. Adams, D. V. Novikov, T. Hiort, G. Materlik, and E. Kossel, Phys. Rev. B 57, 7526 (1998).

${ }^{21}$ S. Marchesini, N. Mannella, C. S. Fadley, M. A. Van Hove, J. J. Bucher, D. K. Shuh, L. Fabris, M. J. Press, M. W. West, W. C. Stolte, and Z. Hussain, Phys. Rev. B 66, 094111 (2002). 